January 2002

\title{
Health-seeking behavior of Karachi women with reproductive tract infections
}

Lubna Ishaq Bhatti

Aga Khan University

Fariyal F. Fikree

Aga Khan University

Follow this and additional works at: https://ecommons.aku.edu/pakistan_fhs_mc_chs_chs Part of the Community Health and Preventive Medicine Commons

\section{Recommended Citation}

Bhatti, L. I., Fikree, F. F. (2002). Health-seeking behavior of Karachi women with reproductive tract infections. Social Science and Medicine, 54(1), 105-117.

Available at: https://ecommons.aku.edu/pakistan_fhs_mc_chs_chs/475 


\title{
Health-seeking behavior of Karachi women with reproductive tract infections
}

\author{
Lubna Ishaq Bhatti, Fariyal F. Fikree* \\ Department of Community Health Sciences, The Aga Khan University, Stadium Road, Karachi 74800, Pakistan
}

\begin{abstract}
To explore the contextual factors influencing health-seeking behavior of women in Karachi regarding reproductive tract infections, 18 women with reproductive tract infections from different clinics and community settings were identified and in-depth interviews were conducted. Physicians in our study diagnosed a woman to have lower reproductive tract infection if she complained of malodorous vaginal discharge with or without perineal itching; and to have pelvic inflammatory disease or upper reproductive tract infection if she had any two of the following complaints: malodorous vaginal discharge, menstrual irregularities, lower abdominal pain or dyspareunia. Women consulted a variety of healthcare providers in their pursuit for treatment, mainly allopathic doctors and hakims. The different treatments prescribed to women ranged from oral and intravaginal medications to various home remedies including refraining from specific foods. Causes of reproductive tract infections reported were "melting bones", consuming foods with perceived hot composition, poor personal hygiene and procedures like dilatation and curettage, delivery and induced abortions. None reported sexually transmitted diseases as the perceived cause of their problem. Interference with religious activities, sexual relationships or socializing was reported as consequences of reproductive tract infections, in addition to lower abdominal pain, menstrual irregularities, backache and kamzori (weakness). Pakistani women seek care for reproductive tract infections and visit a variety of providers, though causes and treatments offered are usually not related to sexually transmitted diseases. We therefore suggest training of healthcare providers for appropriate counseling and that treatment management protocols be advocated. (C) 2001 Elsevier Science Ltd. All rights reserved.
\end{abstract}

Keywords: Reproductive tract infections; Vaginal discharge; Pelvic inflammatory disease; Pakistan; Health-seeking behavior

\section{Introduction}

Reproductive tract infections (RTIs), a global health problem, is a generic term that covers three types of infections: sexually transmitted diseases (STDs), infections that result from overgrowth of organisms normally present in the reproductive tract, and infections associated with medical procedures, including abortions and insertion of intrauterine devices (Tsui, Wasserheit, \& Haaga, 1997). Studies in India, Bangladesh and Egypt

*Corresponding author. Current address: Population Council, One Dag Hammarskjold Plaza, New York 10017, USA. Tel.: + 1-212-339-0605; fax: + 1-212-755-6052.

E-mail address: ffikree@popcouncil.org (F.F. Fikree). have shown that $52-92 \%$ of women are suffering from RTIs (Bang et al., 1989; Singh et al., 1995; Wasserheit, Harris, Chakraborty, Kay, \& Mason, 1989; Younis et al., 1993). Physiological and social factors make women more vulnerable than men to infection with STDs and reproductive tract infections (RTIs). Once infected, it is more difficult to diagnose STDs and RTIs in women as, often, infections occur asymptomatically (Natraj, 1994a). According to a WHO estimate, in 1995, at least 333 million new cases of curable STDs had occurred globally, 150 million of which were in South and Southeast Asia (WHO, 1995).

The significance of lower reproductive tract infections lies not only in morbidity or possible asymptomatic transmission, but also in the propensity for causing 
infection of the upper reproductive tract, i.e. pelvic inflammatory disease or PID (Westrom \& Mardh, 1989). In Asia, 34\% of the PID hospital admissions are a result of RTIs (Brabin, 1993). Although only $10-20 \%$ of RTIs result in PID leading to chronic complications, these episodes increase the risk of tubal infertility, ectopic pregnancy, chronic pain, menstrual irregularities or another episode of PID (Brabin, 1993; Elias, 1991). Furthermore, untreated RTIs are also thought to increase the chances of contracting HIV by three to five times (Natraj, 1994b; Faundes, 1994).

There are no consistent prevalence patterns across countries or even within the same continent to indicate which groups of women are likely to be most in need of services (Westrom \& Mardh 1989). Although women in the general population are considered to be at "low risk", the prevalence of RTIs in this group has been reported to be very similar to high-risk populations (ICWH, 1991). This may be due to high frequency of asymptomatic disease and long duration of infectiousness, as well as to the high-risk behavior of women's spouses (Brabin, 1993; Tsui, Wasserheit, \& Haaga, 1997; Wasserheit \& Holmes, 1992).

RTIs are not just a clinical or epidemiological problem but encompass larger psychosocial ramifications-mental, economic, political, and social factors that are embedded deep in the web of causation (ICWH, 1991). Women in the developing world have little, if any, accurate information on the causes, consequences, and treatment of STDs and related endogenous infections. These problems have devastating personal effects and ultimately lead to social ostracism, and compromised economic and social security (Brabin, 1993; Elias, 1991). The view in many societies is that RTI is a "shameful" illness and, in many cases, dangerous to marriage stability (Elias, 1991; ICWH, 1991).

The existing socio-cultural barriers and taboos associated with diseases even remotely connected with reproduction and sexuality are major hindrances to women seeking help for RTIs (Luthra \& Saxena, 1991). Women initially ignore their symptoms but seek treatment only when their symptoms increase in severity (Kanani, Latha, \& Shah, 1994). In addition, women are reluctant to seek treatment because of the cultural inhibitions and shame felt in consulting male doctors, especially for gynecological disorders. Lack of time and money, as well as the poor availability of health facilities play an important role in delays women incur in seeking healthcare (Bang \& Bang, 1994; Narayan \& Srinivasan, 1994).

Traditional remedies are often the first line of healthcare sought, including some remedies that may introduce or exacerbate infection (ICWH, 1991; Kanani et al., 1994; Olukoya \& Elias, 1996). A survey in Kenya found that Western medical practitioners were consulted for STDs only when herbalists and traditional healers failed to provide relief (Mulder, 1994). When women do seek public or private health services, they can be at high risk of infection if procedures are improperly performed due to lack of sterilized equipment or poor training (ICWH, 1991).

Due to the adverse effects on the reproductive health and well-being of both men and women, RTIs-including STDs have become a major public health concern in developing countries. The emergence and spread of HIV have made the prevention and management of STDs even more important and urgent (ICDDR, B, 1998). However, though RTIs threaten the health of many women, they continue to be ignored by health professionals and research communities in developing countries. There has been little research, and only a few pilot programs have been developed and implemented to evaluate effective strategies to combat RTIs at the primary healthcare level (Whittaker \& Larson, 1996).

This paper is part of a larger study examining women's health-seeking behavior for various gynecological morbidities. We explore herein the contextual factors that influence health-seeking behavior of women from lower socio-economic classes regarding RTIs with special emphasis on the types and treatment modalities offered by various healthcare providers. Furthermore, we also delve into personal discomfort, effects on social and physical health, and the religious ramifications of vaginal discharge.

\section{Methods}

Discussion guidelines were developed and pre-tested on three women in a primary healthcare facility catering to the health needs of women from lower and middle socio-economic classes. Extensive revisions were made in the content of the guidelines as well as in the conduct of the interviews following pre-testing. For example, questions that did not generate any meaningful responses were removed and more direct rather than exploratory questions were added. The interview technique was geared to be active since we found women in the pre-testing phase were replying monosyllabically to most of the questions. At the same time, a conscious effort was made to explore treatment modalities and explanations of disease provided by non-allopathic healthcare providers. In addition, once the actual interviews began, the guidelines were further modified so as to enable more details to be elicited on the themes under study.

We were interested in two categories of RTIs: upper and lower. Lower RTIs (LRTIs) refer to infections in the vulva, vagina or cervix while upper RTIs (URTIs) or PID refers to infection in the uterus, fallopian tubes or ovaries (Younis, Khalil, Zurayk, \& Khattab, 1994). We defined a woman to have a lower RTI if she complained 
of malodorous vaginal discharge, and/or itching or burning sensation in the perineal region. Eligibility criteria for URTI included presence of either two of the following complaints: malodorous vaginal discharge (with or without itching in the perineal region), menstrual irregularities, ${ }^{2}$ lower abdominal pain or pain during sex (dyspareunia). For example, women who reported malodorous vaginal discharge and dyspareunia or menstrual irregularities and lower abdominal pain were considered as PID/URTI.

The interviews were carried out over a period of 7 months (from November 1996 to May 1997) in several locations: a low-income, densely populated urban squatter settlement; the outpatient department of a primary care hospital; and the gynecology clinic of a secondary and a tertiary care hospital. These healthcare facilities cater to populations with socio-economic characteristics similar to the community setting. A balanced ethnic mix was sampled across the various sites. Allopathic doctors or local female health workers from the selected health facilities or community identified women suffering from any type of RTI. These health workers were resident of these communities and knew a lot of these women personally. This not only facilitated easy location but also openness of responses.

The objectives of study, including the need for an ethnic balance, were discussed with the field worker and allopathic doctors at the clinics. They were then asked to refer women with RTIs to the researcher. At the healthcare facilities, women were sometimes approached initially by the interviewer and, after establishing their eligibility, were interviewed prior to being seen by an allopathic doctor. Appointments to return on a mutually convenient day were made with five women who were identified in the clinics and community. Only four of these interviews were conducted as one of these women did not come back to be interviewed. This procedure of selecting our respondents from clinics may have resulted in an over-sampling of women seeking care from allopathic healthcare providers. The interviews were conducted either in separate rooms or in as private an area as possible, nearly always without any other person present. There was only one exception when two nieces of a respondent, alternately, acted as interpreters.

The study objectives were described to respondents before the interviews took place and they were assured of the anonymity of their responses. Verbal consent was obtained for conducting and taping the interviews. Interviews were conducted in the native languages of respondents, which included Urdu, Punjabi and Hindko. The first portion of the interview elicited sociodemographic information in an attempt to create a

\footnotetext{
${ }^{2}$ Menstrual irregularities/disorders include menorrhagia, polymenorrhea, and dysmenorrhea.
}

relaxed atmosphere. The second part dealt with the various healthcare providers sought by these women for this particular problem and the tests and treatments advised by them (Appendix A). Finally, the interview sought to uncover the effects of RTIs on the women's lives, such as personal discomfort and social embarrassment. They were also probed on the perceived effects of RTIs on their physical health and sexual relationships. Although the interviewer used the guideline questions to focus the discussions, the interview was not limited to these questions. In fact, women were further asked to elicit explanations and give additional details. For instance, we initially did not have any questions on religious ramifications of vaginal discharge, but this theme was explored in some interviews as some women raised it during discussions.

We identified a total of 22 women who met our eligibility criteria. One woman, who had made the appointment to come back for the interview, did not return. One woman left at the beginning of the interview, as she felt uncomfortable with the tape recorder and the questions. Two of the interviews were unusable due to the poor quality of the recording. These interviews were considered incomplete and were therefore not included in the analysis. A total of 18 interviews were completed, of which only two were conducted in the community. ${ }^{3}$ Of the six interviews conducted for URTI, three of the women had diagnosed cases of acute PID. ${ }^{4}$ Each interview session lasted anywhere between half an hour to one hour. The interviews were taperecorded, though extensive written notes were also made simultaneously. All these interviews yielded usable transcripts.

The researcher (LIB) transcribed the interviews directly into English to minimize data loss, keeping the original phrases and key words used by the respondents. During the analysis process, we decided to analyze interviews of both types of RTIs together, as most of the findings were similar. The interviews were read several times by both team members to identify the common major themes and a loose coding framework was developed through mind maps. We coded the interviews subsequently and performed content analysis using Ethnograph $\vee 4.0^{\mathbb{R}}$ software package. The analysis methodology included open coding, grouping of findings from these codes and constant comparison of open codes and the categories within the various subgroups.

\footnotetext{
${ }^{3}$ Two interviews in the community, five in the primary care facility, four in the secondary care facility, and seven in the tertiary care facility.

${ }^{4}$ These women had acute lower abdominal pain along with chills and fever, and were diagnosed to have acute PID by their attending gynecologists prior to the interviews.
} 


\section{Results}

\section{Socio-demographic information}

Among the 18 women interviewed, 12 presented with vaginal discharge (LRTI) and the remainder met our criteria for PID (Appendix B). The mean age of women in our sample was $35( \pm 8.6)$ years and, on average, they had been married for $16( \pm 8.9)$ years. The mean monthly income of the household was 5280 Rupees $^{5}$ ( \pm 2556) (Table 1).

\section{Time before seeking care}

On average, women sought care 3 months after the onset of the problem. However, three women sought care in a matter of days due to acute lower abdominal pain, part of our syndromic definition of PID. ${ }^{6}$ Women with PID, who had menstrual disorders like menorrhagia, irregular bleeding or dysmenorrhea as one of their reported symptoms, sought care earlier than those who did not report these menstrual disorders in addition to other symptoms such as malodorous vaginal discharge, lower abdominal pain or dyspareunia.

\section{First contact}

Women generally discussed their signs and symptoms with those they were most intimate with (husbands or mothers-in-law). Previous experience dealing with this problem did not seem to influence the choice of the first person to talk to. A 35-year-old woman stated: I talked to my father-in-law's second wife. She stays at home so I told her. In one instance, it was a cousin who first heard about it. This 38-year-old woman reported: I talked to my distant cousin; I talked to her first of all. My husband does not like to talk about these issues. Even now, I had to beg him to come to the hospital.

\section{Terms used to describe vaginal discharge}

Women tended to use similar terms to describe vaginal discharge or symptoms of PID. This may be attributed to our selection bias toward urban women or the medicalization of the problem. Many women in our sample used the medical term "leucorrhea". Our findings suggest that women, in their quest for treatment, pick up their vocabulary from different healthcare providers or other women who may or may not be suffering from a similar problem.

\footnotetext{
${ }^{5} \mathrm{US} \$ 1=40$ Rupees.

${ }^{6} \mathrm{An}$ acute episode of PID is defined as fever and chills accompanied by lower abdominal pain.
}

Table 1

Frequency distribution of socio-demographic characteristics of 18 women interviewed

\begin{tabular}{|c|c|}
\hline Sociodemographic factors & $\begin{array}{l}\text { Number of } \\
\text { women interviewed }\end{array}$ \\
\hline \multicolumn{2}{|l|}{ Current age (years) } \\
\hline $21-30$ & 2 \\
\hline $31-40$ & 11 \\
\hline $41-50$ & 2 \\
\hline$>50$ & 1 \\
\hline $\mathrm{NA}^{\mathrm{a}}$ & 2 \\
\hline \multicolumn{2}{|l|}{ Occupation } \\
\hline Gainfully employed & 3 \\
\hline Housewives & 15 \\
\hline \multicolumn{2}{|l|}{ Education } \\
\hline Illiterate & 3 \\
\hline$\leqslant 5^{\mathrm{b}}$ & 3 \\
\hline $6-10$ & 3 \\
\hline $10-14$ & 2 \\
\hline $\mathrm{NA}^{\mathrm{a}}$ & 7 \\
\hline \multicolumn{2}{|l|}{ Ethnicity } \\
\hline Pathan $^{\mathrm{c}}$ & 4 \\
\hline Punjabi $^{\mathrm{d}}$ & 6 \\
\hline Mohajir ${ }^{\mathrm{e}}$ & 8 \\
\hline \multicolumn{2}{|l|}{ Family system } \\
\hline Nuclear & 6 \\
\hline Extended & 12 \\
\hline \multicolumn{2}{|l|}{ No. of children } \\
\hline 0 & 3 \\
\hline $1-2$ & 4 \\
\hline $3-5$ & 8 \\
\hline$>5$ & 3 \\
\hline
\end{tabular}

${ }^{\mathrm{a}}$ Information not available.

${ }^{\mathrm{b}}$ Years of schooling.

${ }^{\mathrm{c}}$ Pathan Community - main language is Pushto and generally come from the North West Frontier Province.

${ }^{\mathrm{d}}$ Punjabi Community-main language is Punjabi and generally come from Punjab Province.

${ }^{\mathrm{e}}$ Mohajir Community-main language is Urdu and represents those who migrated from India at the time of Independence (1947). Mostly settled in Karachi.

Q. What is this particular illness called in your language?

A. It is called leucorrhea. Doctors call it leucorrhea.

Q. Where have you heard of this term?

A. From doctors. The homeopathic doctors call it leucorrhea as well. I have heard it from them.

(24-year-old woman)

Although we probed at the relationship between the severity of the disease and the terms used, we were unable to elicit any association (Table 2). 
Table 2

Glossary of terms for vaginal discharge and pelvic inflammatory disease reported by the interviewees

\begin{tabular}{lll}
\hline Type of RTI & Terms & Translation \\
\hline Vaginal discharge & Leucorrhea & Vaginal discharge \\
Mawad & Pus (vaginal discharge) \\
Pani ki shikaet & Pani painda paya hai & Problem of water (vaginal discharge) \\
Pani jaana & Water is coming out (vaginal discharge) \\
Chita pani painda paya hay & Water going (vaginal discharge) \\
& Pait ka dard & White water is coming (white vaginal discharge) \\
Naloon mein peep par jana & Pachaydani mein peep parna \\
Pait dukhe say & Pus in the fallopian tubes \\
& Naf ke nechay dard & Pus in the uterus \\
Pani pur raha hay & Stomach hurts (lower abdominal pain) \\
Nalon ka dard & Pain below the umbilicus (lower abdominal pain) \\
& Water is coming (vaginal discharge)
\end{tabular}

\section{Reported causes of vaginal discharge and PID}

The reported causes of RTIs ranged from different types of food ingested to medical causes. Respondents either perceived these or were told by various healthcare providers or by relatives/friends. A 24-year-old woman unveiled a very interesting phenomenon. Her allopathic doctor told her that ... when the body is weak, then water from the bone starts to flow and that is discharge. Weakness, like the one that you have after the children, that's why you start having discharge.

Other women, or in some cases healthcare providers, advised women suffering from RTIs that this was a consequence of consuming specific categories of food. We believe that some of these concepts are borrowed from Schools of Eastern Medicine where emphasis is on the importance of temperament, body fluids, and foods (Zaman, 1996; Al-Haziq (2nd edition) and Akseer-eAzam).

Now if I eat rice or anything with hot composition, like chicken, red meat or masoor (a type of black round lentil very common in the subcontinent), then I get discharge. Then I went to Dr. Sohrab's ${ }^{7}$ clinic. She said that it (discharge) occurs because of eating potatoes and rice.

(46-year-old woman)

My mother-in-law said that you don't take milk. You should drink milk regularly. That's why this happens. Then somebody said to eat bananas. Hence I did all these gharailoo ilaj (home remedies) that people told me about. I thought that maybe doing these things

\footnotetext{
${ }^{7}$ Names of doctors and hospitals/clinics have been changed.
}

would cure this problem, because the doctors don't tell me the reason behind it.

(32-year-old woman)

Inadequate personal hygiene is known to increase the risk for reproductive tract infections (Naguib, Nagui, Bassaly, \& Gaber, 1975; Younis et al., 1993). Two women mentioned hygiene as a probable cause of RTIs.

My mother said that you live in a dirty place like Korangi. There is so much dust and the water is so dirty, you must have got this infection that way.

(32-year-old woman)

You know the cloth that I use for menses, I use a washed cloth and I even boil it. I sometimes think that maybe I got this infection because of this.

(25-year-old woman)

Women also related their symptoms to different procedures or conditions that they had undergone. Two women attributed their LRTI to their pregnancies:

It happened after my third child was born. Right after his delivery. When I took a bath after sawamahina ${ }^{8}$ then the discharge started.

(24-year-old woman)

One 38-year-old woman attributed her LRTI to tubal ligation:

Actually I had tubal ligation two years back. The discharge increased after that but now for six months

\footnotetext{
${ }^{8}$ An Islamic religious ritual after childbirth. Although women wash and bathe regularly during the postpartum period, the 40th day postpartum is observed with a ceremonial bath, after which prayers and sexual intercourse are permitted.
} 
it is coming in pieces just like froth or sputum. It is foul-smelling as well.

A 46-year-old woman blamed her induced abortion for the vaginal discharge.

It is because of the induced abortion that I had. Because of that, I think that there is some defect or some wound has developed. It is because at that time they have used ozar (instruments).

Not having adequate information about the causes or consequences of RTIs also lead to anxiety, as expressed by this 32-year-old:

I am scared about whether this is a dangerous disease or not. I have told the doctors but they don't understand anything. I think it is due to the ring (IUCD) that I had put in. People say different things, that you get AIDS by this.

Although, the cause of many of these infections may be sexually transmitted by spouses, none of the women or, for that matter, any of the healthcare providers visited by these women, suggested sexual transmission as a possible causative factor for RTIs. The role of men in the spread and increased prevalence of RTIs in the conservative communities of developing nations such as Pakistan is being disregarded.

\section{Choice of healthcare providers}

The women in our study sample consulted a wide range of healthcare providers. On average, a woman went to three to four healthcare providers - mainly family practitioners, gynecologists and traditional birth attendants (TBAs). Their choice of a particular provider was based on recommendations from friends, relatives or social contacts who had been successfully treated for vaginal discharge or any other gynecological problem.

I talked to my husband first, then on his suggestion talked to my mother-in-law. They both said to go to a (allopathic) doctor. My husband's sisterin-law (his brother's wife) goes for her treatment to Dr. Shaiza, so my mother-in-law took me there. Then she wrote a letter for Dr. Ruqiya here and I came here.

(20-year-old woman)

The first provider was almost always an allopathic doctor except in two cases where treatment was initially taken from a dai (TBA) and a homeopathic doctor, respectively. Women were final arbiters in deciding where to seek treatment, except in two cases where their mothers-in-law made the final decision, and these women reported accepting this. A 32-year-old woman said:

I went to Baqai hospital. Actually my sister was admitted there. There my brother-in-law (he is an allopathic doctor) said that you should present yourself. So I just made the payment there, and presented myself in that hospital.

Although their husbands, mothers-in-law, family members or neighbors accompanied women when they went to consult a healthcare provider, a majority of women went to visit the providers alone. One woman stated that her husband did not allow her to go out of the house and her high blood pressure was the only reason that she was able to seek treatment for her PID.

Money or the cost of treatment was not a criterion to choose a particular provider for women interviewed except for this 46-year-old woman:

I save money from household expenses and then use that money. My husband does not let me spend money on my treatment. He sometimes gives me some money for the house. That's why I do the treatment sometimes and then leave it.

Generally, the reasons reported by women for changing healthcare providers were not obtaining relief, referral by one provider to another, cumbersome treatment protocols and changing over to a female provider from a male one.

Q. Why and when did you change over to the other providers?

A. He is a male doctor. He just gave me medications. He told me to go to a lady doctor (gynecologist). He said go to any hospital you want to but go to a lady doctor (gynecologist) and have a check-up (vaginal examination). I came to this hospital because my husband's sister comes here all the time and so we know the hospital.

(35-year-old woman)

\section{Allopathic doctors}

All 18 women interviewed for RTIs visited allopathic doctors at least once in their quest for treatment. Women identified these allopathic doctors either through their relatives, or through previous experience with these providers. Women in our sample consulted many allopathic doctors, varying from family practitioners in their localities to qualified gynecologists in tertiary care hospitals. Allopathic doctors asked details about the discharge and generally tended to conduct a pelvic examination. Although women reported that the pelvic examination was an unpleasant procedure, a majority of them said that it was not 
Table 3

Reported causes of vaginal discharge and pelvic inflammatory disease

\begin{tabular}{|c|c|c|c|}
\hline Type & Cause & Translation & Source \\
\hline \multirow[t]{10}{*}{ Vaginal discharge } & Pakhaney ke keeray & Worms in stool & Allopathic doctor \\
\hline & Jisim ki kamzori & Weakness in body & Allopathic doctor \\
\hline & Hadion ka pani & Water of bones & Homeopathic doctor \\
\hline & Gandgi & Dirt & Allopathic doctor \\
\hline & Buri Qismat & Bad luck & Self \\
\hline & Mahiney ke liye kapra istamaal kurna & Using cloth for menses & Self \\
\hline & Aloo chawal Khana & Eating potatoes and rice & Allopathic doctor \\
\hline & Under zakham hona & Having wounds inside vagina & Allopathic doctor \\
\hline & Under infection hona & Having infection inside vagina & Allopathic doctor \\
\hline & Qudrat ki marzi & Nature's will & Self \\
\hline \multirow[t]{3}{*}{ Pelvic inflammatory disease } & Rasoli & Mass/ tumor & Allopathic doctor \\
\hline & Qudrat chahti thee & Nature wanted so & Self \\
\hline & Bachaydani ke moon per zakham & Wound on the opening of cervix & Allopathic doctor \\
\hline
\end{tabular}

painful. A 45-year-old woman describing her experience of undergoing a vaginal examination said:

They had used an instrument and taken some samples. I was uncomfortable and I did tell the doctor. She said that it was necessary and then she told me to relax.

Only three allopathic doctors asked for any laboratory procedures or tests: in one case, an abdominal ultrasound, when the woman complained of lower abdominal pain, and a culture of the vaginal discharge in the other two.

The different treatments that were prescribed to women by allopathic doctors were tablets, syrups, injections, vaginal creams and pessaries. ${ }^{9}$ Women did not know the names of the tablets and pills as these were mostly distributed in paper sachets or the names were difficult to remember. However, two women mentioned Flagyl ${ }^{\circledR 10}$ - a recommended therapy for bacterial vaginosis. One woman reported being prescribed calcium tablets by an allopathic doctor. This was the same woman who reported "melting bones" as the cause of her LRTI.

While allopathic doctors prescribed vaginal creams and pessaries, they did not counsel their patients on its appropriate use. As a result, these treatments became cumbersome and women faced problems in following instructions appropriately. A 24-year-old woman stated:

You know the doctor gave me these very big syringes to fill with the cream and put that in the vagina. But when I used to go to the washroom in the mornings,

\footnotetext{
${ }^{9}$ For fungal and non-specific bacterial vaginal infections.

${ }^{10}$ Brand name for metronidazole.
}

all the dawa (medication) would come out. All of it would come out. Nothing would remain in.

Only one of the allopathic doctors asked for a dilation and curettage (D\&C) but we could not elicit from the woman the reason why it was ordered. Allopathic doctors were the only healthcare providers in our sample who referred women to gynecologists or to tertiary care hospitals for further investigations and treatment.

Some allopathic doctors gave obscure reasons to women for these problems (see Table 3), such as eating potatoes and rice; worms in the stool leading to infection in the vagina or weakness of body resulting in water flow from bones, observed as vaginal discharge. These notions and their relationship to RTIs are not mentioned anywhere, to our knowledge, in the medical literature.

\section{Dais or traditional birth attendants}

Only two women in our sample consulted a dai. One woman remarked that, as her husband did not allow her to make friends, she was unaware of any TBAs in her locality and hence never consulted one. Two women reported seeking treatment from dais earlier during their pregnancies and they consulted them again for RTIs but they did not take the treatment the dais had prescribed because their mothers-in-law forbade them. A 24-yearold woman said:

I went to this dai near my mother's house about one week back. She said that she would give me something to eat. She will also put some medication in the vagina as well. I told my mother-in-law and she said "don't go for this kind of treatment. Go to a doctor (allopathic)". 
A 46-year-old woman described the treatment that a dai gave her:

I went to this dai near my house. She used to put this medication in my vagina. She used to immerse cotton balls in a dark reddish or maroonish liquid and put that in my vagina. She used to put it in and take it out herself.

\section{Hakims}

Only five women consulted hakims (physician in India and Muslim countries). Sources of referral were husbands, friends and relatives. Hakims never conducted a pelvic examination or recommended any laboratory tests. A majority of the hakims gave safoof (powders), khameeray (thick syrups), ma'jun (an intoxicating drug prepared from hemp extract and sugar), and joshandah (a concoction that is boiled to extract the essence from certain medicinal herbs). One hakim also advised a woman to take oral medication while refraining from consuming beef, rice, and badi cheezein (flatulent foods) resulting in the woman discontinuing the treatment, as she could not consume these foods. Two respondents said that with hakim's medication, their bodies became edematous and they had to discontinue the treatment.

One 35-year-old woman said that apart from the oral medications, she was also given some powder to cook in water at home and bring to the clinic for further procedure. She said:

They gave me a joshandah (a concoction that is boiled to extract the essence from certain medicinal herbs) to cook at home. Then when I took it there, they washed the inside of my vagina and the outsides. Then they put some marham (paste of medicinal herbs) inside me. They told me that I have a lot of waram (swelling).

\section{Homeopathic doctors}

Only two women went to consult homeopathic doctors, though both were displeased with the treatment recommended. For example, a 24-year-old woman reported that when she had developed vaginal discharge during her first pregnancy, she decided to opt for homeopathic medication. Her problem was relieved for the first two pregnancies, but then she could no longer comply with the laborious treatment. She said:

The homeopathic doctor tells me to refrain from many things, like don't eat this, don't eat that, like don't take garam cheezein (things with hot composition), fish, egg, chicken, beef. These things were forbidden for me to consume. He asked me to stop eating all this. Now if I stop eating all this then, what will remain in my body? Lentils and vegetables. So I stopped the homeopathic treatment as well.

\section{Spiritual healers}

Only one woman was referred by her mother-in-law to a spiritual healer. At one place, she was given a taweez (amulet), which did not help her. Describing her visit to second place, this 28-year-old woman said:

I went to this big religious person who sits in North Karachi. His female assistant saw me and then she said that there is nothing. What you have to do is take eggshells, boil them in water, and then take the membrane out. Then grind the shell and make a powder. Take a pinch of this powder every day in the morning and one in the evening. I did that even. But nothing happened with that either. It stopped for a few days and then it started again.

\section{Home remedies}

Women in our sample were told of many home remedies either by their relatives or friends. Some of these were:

Somebody told me that to eat two bananas and take milk on top of that before sleeping. It will cure your problem $100 \%$. But nothing happened. It stops for a few days and then it starts again.

(35-year-old woman)

I have eaten khopra (coconut). You can eat it either fresh unripe or the completely dry way. You eat thandi cheezein (things with cold composition). You know, like cucumber and sugar cane juice. Someone also told me that you have to take the skin of pomegranate. Soak it overnight in cold water and in the morning drink the strained water before having anything else.

(46-year-old woman)

\section{Effects of RTIs on women's well-being}

RTIs can compromise a woman' physical and/or social well-being. Our data shows that women report lower abdominal pain, menstrual irregularities, backache, kamzori (weakness) and, on a social level, interference with religious activities, sexual relationships or socializing as consequences of RTIs.

\section{Physical health}

Eleven women complained that, as a result of vaginal discharge, they had developed lower abdominal pain, backache and weakness, which affected their general sense of well-being. Furthermore, inclusion of lower 
abdominal pain and menstrual irregularities (menorrhagia and dysmenorrhea) as symptoms of vaginal discharge further exacerbated their ill health. Discussions with women who reported these additional complaints suggest that these problems contributed significantly to their overall burden of ill health.

Then I started getting mawad (pus). Then I took a lot of treatment for this but I didn't get relief anywhere. Now my periods are irregular and it comes very little. I get a lot of pain in my legs. I get a lot of pain in my back as well. I feel inside as if someone is cutting my flesh. And the color of my bleeding has also changed. (38-year-old woman)

It is very serious for me because I am having pain. It makes my work difficult. I was not able to work initially. But then I started working so I won't feel the pain that much. It is difficult but if you are busy then you feel the pain a little less.

(20-year-old woman)

Of the 18 women interviewed, seven women complained that they experienced dyspareunia. ${ }^{11}$ There were various reactions from their spouses: three husbands promptly refrained from sexual intercourse, while one 38-year-old woman stated that: It hurts during sex but when I tell my husband, he says that tum drama kar rahee ho (you are dramatizing) and you are not feeling any pain. He is a Pathan and Pathans do not care about their women.

Six women complained of recurrent vaginal discharge despite taking various treatments.

Then I went to this hospital. They gave me dawa (medications) to eat. When I was taking those, I was all right. When I stopped taking the dawa (medication), then the problem started again. So I took the dawa (medication) again from there. I was all right when I was taking the dawa (medication) and then when I stopped this time, then the problem started again. Then I did not go back there. The problem kept on happening and it is still happening.

(24-year-old woman)

Then I went to another doctor (allopathic) and she gave me same tablets to keep in my vagina and some cream as well. When I took that tablet then it helped and the discharge stopped. But then now, after one month, it has started again.

(34-year-old woman)

\section{Religious ramifications}

Our data suggested that vaginal discharge did interfere with the religious obligations of the 18 Muslim women. According to the religious tenets of Islam, cleanliness prior to each of the five prayers is

\footnotetext{
${ }^{11}$ Pain during sexual intercourse.
}

"mandatory", and women experiencing vaginal discharge would not be considered "clean". ${ }^{12}$. Women in our sample generally commented that they rinse their perineum and change clothes prior to each of the mandatory five daily prayers. A 46-year-old woman said:

I have a lot of problems whenever I want to say my prayers. I change shalwar (pants) every time I want to say my prayers. I have kept a separate shalwar (pants) for prayers. I change into that before my prayers and then change back.

Two women asked religious leaders for guidance in this problem.

No, I say my prayers. I just go and wash myself. And then say my prayers. Then again when I have to say my prayers, then I again go and wash. I just wash almost the whole day.

(35-year-old woman)

A 24-year-old woman stated that she started saying her prayers irregularly because of vaginal discharge.

I have many problems. It is so much that I have to change my shalwar (pants) two, three times in a day to say my Namaz (prayers). I was saying my prayers till the day before yesterday, and then the discharge started again. Now I have stopped saying my prayers.

\section{Social health}

Our data suggests that RTIs hinders women in their socializing either due to kamzori (weakness) or to mehfil mein na baith sakna (inability to sit in social gatherings). For example, a 46-year-old woman stated:

Now as the time is passing and the age is increasing, you know you have to go back to your village to meet family and relatives. The routes are very long. This problem gives me a lot of difficulties. Now I cannot even walk to this bus stop near my house.

Problems associated with sitting in social gatherings are aptly described by a few of the women:

Itching is bad. I get scratches on my inner thighs and then I get burning sensation down there. Now I have developed wounds there. I have problem walking because my wounds rub against each other.

(45-year-old woman)

The discharge started first, then the itch started in my perineum. It started to itch so badly that I was not able to sit in front of the guests.

(24-year-old woman)

\footnotetext{
${ }^{12}$ Bahishti Zaiwar (1981), Maulana Mohammed Ashraf Ali Thanavi, Daraul-Ashaat, Karachi.
} 
Believe me, the smell is so bad that I cannot tolerate that myself. Sometimes, I don't take my meals for two, three times because of this smell, this stench.... The truth is that when I am sitting with someone, I can smell the odour. Then I think that if I can smell this, then the next person must be able to smell it too. (32-year-old woman)

Women with RTIs felt stigmatized by these problems. A woman described the emanation of vaginal discharge as being a cause of her low self-esteem:

I was unable to understand what this whole thing is. And whenever I was about to have sex with my husband, I used to wash myself thoroughly because if he sees it he would say 'what kind of woman is my wife that something is coming out of her?' I was getting this inferiority complex day by day but I was unable to understand what this discharge is.

(32-year-old woman)

\section{Discussion and conclusion}

Women in our sample pursued treatment for RTIs and ceaselessly visited various types of healthcare providers. These women belonged to an urban population and this may have influenced the health-seeking behavior pattern of invariably consulting an allopathic doctor first. However, after the initial contact, the type of healthcare provider subsequently consulted varied, and women also consulted providers from alternative health systems, such as hakims, dais, homeopathic doctors and spiritual healers. For example, although dais conduct the majority of deliveries in Pakistan (PDHS, 1992), only two women in our sample consulted dais and only one used the treatment recommended. A possible explanation may be that dais are considered to be the providers of obstetric care only. Interestingly, many family members did not approve of providers from the alternative healthcare system for pursuing a course of treatment for vaginal discharge. This lack of support from the patient's social network may have also contributed to consulting allopathic doctors initially and reverting to other types of providers only when dissatisfaction with the treatment provided by allopathic doctors occurred. We feel that several factors may lead to women emphasizing allopathic treatment-the location of most of the interviews, the medical background of the researcher, or possibly a reluctance to mention other styles of treatment.

The majority of women consulted female healthcare providers. Some of the male providers initially consulted referred these women to female allopathic doctors, as they could not perform vaginal examination, mainly because of cultural inhibitions. Our results support findings by Bang and Bang (1994) and Narayan and Srinivasan (1994) regarding reluctance and shame felt by women when consulting male doctors for gynecological disorders, as a result of socio-cultural inhibitions.

The results from our in-depth interviews illustrate that only few gynecologists from tertiary care hospitals requested laboratory investigations. In addition, when allopathic doctors prescribed treatment regimens like vaginal creams and pessaries, they generally did not counsel their patients on their correct use. Lack of appropriate counseling may reflect time constraints and over-crowded clinics, though this often leads to difficulties faced by women in complying with the duration of the regimens. For example, women did not comprehend why the medication was "all coming out of the vagina in the morning", as the women related "effectiveness of treatment" to complete absorption of medication. This lead to dissatisfaction regarding the effectiveness of treatment recommended and consequently diminished the credibility of the healthcare provider, which ultimately culminated in discontinuation of the treatment. We therefore suggest that healthcare providers should be trained to provide appropriate counseling regarding the use of medications, to encourage the patient's compliance with the treatment.

Women were offered several causes for RTIs. For example, an allopathic doctor told one woman that vaginal discharge is actually "water from melting bones" due to "weakness" and to take calcium tablets to counter this problem. This kind of incorrect knowledge about the causes of RTIs results in inappropriate treatment, which contributes to a longer duration of the infections and serious consequences, such as PID and infertility (Brabin, 1993; Elias, 1991).

The literature has shown that procedures conducted in sub-optimal conditions lead to an increased risk of infections (Luthra \& Saxena, 1991; Wasserheit \& Holmes, 1992). Women in our sample also associated different operative procedures like D\&C, induced abortion and deliveries with their RTIs. It is possible that these women were infected during these procedures due to sub-optimal conditions. We therefore suggest that training of healthcare providers-especially allopathic doctors and dais - to conduct obstetric and gynecological procedures under sterile conditions will decrease the prevalence of iatrogenically introduced infections.

Women complained of numerous other physical symptoms like backache and weakness that they perceived to have resulted as a consequence of an RTI. These additional problems contributed significantly to the burden of disease for these women. Furthermore, half of the women complained of dyspareunia, which affected their sex life. None of the healthcare providers (whether male or female) paid any attention to these 
effects of RTIs and hence these women kept on suffering silently. Physical symptoms of RTIs along with the above-mentioned associated symptoms contributed significantly to the mental health of women. Women were unable to attend social gatherings or travel short distances because of these symptoms, which lead to considerable social restriction. Women were not offered any valid causes for RTIs, which may have added to the stigma surrounding RTIs, as in the case of a woman who reported that her vaginal discharge resulted in low self-esteem. Furthermore, women suffered from religious restrictions imposed by the Islamic requirement of cleanliness prior to each of the five daily prayers. Women in our sample had to wash themselves and change their clothes repetitively in order to pray. At times, women did not say their prayers because they felt "dirty". We feel that this added to the mental stress affecting women in their day-today activities. Lack of appropriate counseling by the healthcare providers on the physical, mental and social burden of ill-health consequent to the vaginal discharge supports our recommendation for training in the area of inter-personal skills-enabling healthcare providers to be more empathetic toward their clients.

Gender inequality must be highlighted in any discussion of communicable diseases, especially sexually transmitted infections. Results of our study highlight this issue both from the providers' and the women's perspective. One might understand the persistent view that RTIs are "shameful" as constructions that protect male partners from responsibility for their wives' infections. Furthermore, these interpretations also add to the notion that the women are solely responsible for their infections, overlooking their male partners as possible contributors. Gender exercises an important influence on healthcare providers' perceptions of male or female clients' needs and on the manner and substance of the medical advice they provide to male and female clients. Sexually transmitted diseases are mainly caused by risky sexual practices between sexual partners (Luthra \& Saxena, 1991). The results from our study imply that healthcare providers were unaware of the causes of RTIs and consequently, the lack of knowledge possibly affected the treatment prescribed. Six women complained of recurrent vaginal discharge that may have resulted from re-infection by their sexual partners, though interestingly none of the providers consulted prescribed treatment for spouses suggesting that reinfection by sexual contact was not being considered as a possible cause of re-infection. The lack of emphasis on a male partner's role in the transmission of infection reflects gender inequality of health in a traditional society where definitions of health status and traditional medical practices are indicative of the subordinate social status of women.
Our sample consisted of married women and as a result, we could not include the perceptions of young unmarried girls suffering from vaginal discharge. Furthermore, as the majority of our subjects were identified in healthcare facilities, we feel that our results typify the more "medicalized" picture than if our sample selection had been community based. This is because we may not have identified women who either could not afford treatment or were not allowed to seek care. Finally, our finding of allopathic doctors being the mainstay of the treatment may also reflect the bias in our sample selection mode.

RTIs exert a great burden on the health and wellbeing of women. The results of this study indicate that Pakistani healthcare providers need to be trained with emphasis not only on appropriate management and treatment protocols for RTIs, but also on prevention and appropriate couple counseling. Furthermore, dais also need to be trained on aseptic methods of delivery. Finally, we suggest that further studies be conducted to explore the obstacles that women face in accessing health services for RTIs and providers' depth of knowledge on RTIs.

\section{Acknowledgements}

We would like to express our gratitude to Ms Martine Collumbien and Dr. John Cleland for their intellectual input and invaluable advice. We are also indebted to the doctors and the field worker who helped us during the fieldwork. The study was funded by the DFID under Operations Research component of Population III project. The views expressed are those of the researchers and DFID takes no responsibility for the contents of the paper and views expressed.

\section{Appendix A}

The definitions of different health care providers is given in Table 4.

\section{Appendix B}

The disease classification using syndromic approach is given in Table 5.

\section{References}

Al-Haziq (2nd edition) and Akseer-e-Azam. The books of Hikmat. 
Table 4

Allopathic doctors

Traditional birth attendants (TBA) or dais

Hakims

Homeopathic doctor

Spiritual healers as Baba, Rohani Ilaj, Pirs, Maulvis or Bhuton

Table 5

\begin{tabular}{|c|c|c|c|c|c|}
\hline Number of women & Disease classification & Vaginal discharge & Dyspareunia & Low abdominal pain & Menstrual disorders 3 \\
\hline 3 & $\mathrm{PID}^{\mathrm{a}} / \mathrm{URTI}$ & Yes & - & $\mathrm{Yes}^{\mathrm{b}}$ & - \\
\hline 1 & PID/URTI & Yes & - & Yes & $\mathrm{Yes}^{\mathrm{c}}$ \\
\hline 2 & PID/URTI & Yes & - & Yes & Yes $^{\mathrm{d}}$ \\
\hline 2 & LRTI/Vaginal Discharge & $\mathrm{Yes}^{\mathrm{e}}$ & - & Yes & - \\
\hline 2 & LRTI/Vaginal Discharge & Yes & - & - & Yes $^{\mathrm{f}}$ \\
\hline 5 & LRTI/Vaginal Discharge & $\mathrm{Yes}^{\mathrm{g}}$ & - & - & - \\
\hline 3 & LRTI/Vaginal Discharge & Yes & Yes & - & - \\
\hline
\end{tabular}

${ }^{\text {a }}$ Gynecologists diagnosed as acute PID.

${ }^{\mathrm{b}}$ Acute abdominal pain with fever.

${ }^{\mathrm{c}}$ Irregular bleeding + dysmenorrhea

${ }^{\mathrm{d}}$ Menorrhagia

${ }^{\mathrm{e}}$ Malodorous

${ }^{\mathrm{f}}$ Dysmenorrhea

${ }^{\mathrm{g}}$ White, malodorous, with/without perineal itching.
Those who have completed their medical education from a certified medical college with, at minimum, an MBBS degree, though some may have additional training in obstetrics and gynecology. These doctors are either attached to a hospital or are running one-room clinics in the community as general practitioners.

A person (usually a woman) who assists mothers at childbirth, and who initially acquired skills delivering babies by herself or by working with other Dais $^{\mathrm{a}}$.

Traditional healers, who emphasize the importance of temperaHakims usually prescribe herbal medicines, a strict diet, and often a recommendation to change lifestyle. In most cases, treatment involves rubbing with some balms or the application of herbal drug $^{\mathrm{b}}$.

A person, who practices homeopathy, a system of medical practice according to which diseases are treated by the administration (usually in minute doses) of drugs that would produce, in a healthy person, symptoms like those of the disease treated ${ }^{\mathrm{c}}$.

A person who provides treatment either by using religious verses or calling upon spirits. These spiritual healers are available to men and women of all socio-economic classes. ment and body fluids, and derive their medicines from herbs.

\footnotetext{
${ }^{a}$ Verderese, M. and Turball, L. The Traditional Birth Attendant: A guide to her training and utilization. WHO offset publication.

${ }^{\mathrm{b}}$ Zaman, R. Hakims and Vaids the most popular doctors in Bangladesh, but they know little about AIDS. Aids Analysis Asia. 1996 n-Feb; 2(1): 12-3.

${ }^{\mathrm{c}}$ The Shorter Oxford English Dictionary on Historical Principles. Oxford University Press 1990.
} 
Bang, R., \& Bang, A. (1994). Women's perceptions of white vaginal discharge: Ethnographic data from rural Maharashtra. In J. Gittelsohn, M.E. Bentley, P.J. Pelto, M. Nag, S. Pachauri, A. D. Harrison, \& L.T. Landman (Eds.), Listening to women talk about their health: Issues and evidence from India. New Delhi, India: Har-Anand Publications.

Bang, R. A., Bang, A. T., Baitule, M., Choudhary, Y., Sarmukaddam, S., \& Tale, O. (1989). High prevalence of gynecological diseases in rural Indian women. Lancet, 1, 85-87.

Brabin, L. (1993). Pelvic inflammatory disease. Africa Health, $15,15-17$.

Elias, C. (1991). Sexually transmitted diseases and the reproductive health of women in developing countries. Reproductive health training materials. Working papers No. 5, Population Council, New York.

Faundes, A. (1994). Reproductive tract infections. International Journal of Gynecology and Obstetrics, 46 (2), 181-17.

International Centre for Diarrhoeal Disease Research, Bangladesh (ICDDR,B). (1998). Strategies to improve the prevention and management of RTIs and STDs in Bangladesh. Intervention Update, 3(4), 1-2.

International Women's Health Coalition (ICWH). (1991). Reproductive tract infections in women in the third world: National and international policy implications. Papers from Bellagio Conference, Italy.

Kanani, S., Latha, K., \& Shah, M. (1994). Application of qualitative methodologies to investigate perceptions of women and health practitioners regarding women's health disorders in Baroda slums. In J. Gittelsohn, M. E. Bentley, P. J. Pelto, M. Nag, S. Pachauri, A. D. Harrison, \& L. T. Landman (Eds.), Listening to women talk about their health: Issues and evidence from India. New Delhi, India: HarAnand Publications.

Luthra, U. K., \& Saxena, B. N. (1991). Reproductive tract infections in India: The need for Comprehensive reproductive health policy and programs. Papers presented at Bellagio conference, International Women's Health Coalition.

Mulder, D. W. (1994). Disease perception and health-seeking behaviour for sexually transmitted diseases. In Prevention and management of sexually transmitted diseases in eastern and southern Africa: Current approaches and future directions. Compiled by Network of AIDS Researchers of Eastern and Southern Africa (NARESA), Nairobi, Kenya.

Naguib, Y. A., Nagui, A., Bassaly, M., \& Gaber, A. (1975). Treatment of trichomonas vaginitis with a single dose of tinidazole. Journal of the Egyptian Medical Association, 58, 633-643.

Narayan, N., \& Srinivasan, S. (1994). Some experiences in the rapid assessment of women's perceptions of illness in rural and urban areas of Tamil Nadu. In J. Gittelsohn, M. E. Bentley, P. J. Pelto, M. Nag, S. Pachauri, A. D. Harrison, \& L. T. Landman (Eds.), Listening to women talk about their health: Issues and evidence from India. New Delhi, India: Har-Anand Publications.
Natraj, S. (1994a). The magnitude of neglect: Women and sexually transmitted diseases in India. In J. Mirsky, M. Radlett, W. Davies, \& O. Bennett (Eds.), Private decisions, public debate: Women, reproduction and population. London, England: Panos Publications.

Nataraj, S. (1994b). Waiting for a connection. Popli, 21(10), 6-11.

Olukoya, A. A., \& Elias, C. (1996). Perceptions of reproductive tract morbidity among Nigerian women and men. Reproductive Health Matters, 7, 56-65.

Pakistan Demographic Health Survey (PDHS), 1990-91. (1992). National institute of population studies, Islamabad, Pakistan. Columbia, Maryland USA: IRD/Macro International Inc.

Singh, V., Gupta, M. M., Satyanarayana, L., Parashari, A., Sehgal, A., Chattopadhya, D., \& Sodhani, P. (1995). Association between reproductive tract infections and cervical inflammatory epithelial changes. Sexually Transmitted Diseases, 22, 25-30.

Tsui, A. O., Wasserheit, J. N., \& Haaga, J. G. (1997). Reproductive health in developing countries: Expanding dimensions, building solutions. Washington, DC: National Academy Press.

Wasserheit, J. N., Harris, J. R., Chakraborty, J., Kay, B. A., \& Mason, K. J. (1989). Reproductive tract infections in a family planning population in rural Bangladesh: A neglected opportunity to promote maternal child health family planning programs. Studies in Family Planning, 20, 69-80.

Wasserheit, J., \& Holmes, K. (1992). Reproductive tract infections: Challenges for international health policy, programs and research. In A. Germain, K. Holmes, P. Piot, \& J. N. Wasserheit, (Eds.) Reproductive tract infections: Global impact and priorities for Women's Reproductive Health. New York: Plenum Press.

Westrom, L., \& Mardh, P. A. (1989). Acute pelvic inflammatory disease. In K.K. Holmes et al. (Eds.), Sexually transmitted diseases. New York, USA: McGraw-Hill.

Whittaker, M., \& Larson, A. (1996). Reproductive tract infections: The forgotten and neglected component of family planning services. Venereology, 9, 40-47.

WHO. (1995). An overview of selected curable sexually transmitted diseases. Unpublished paper, WHO Global Programme on AIDS, Geneva, Switzerland.

Younis, N., Khalil, K., Zurayk, H., \& Khattab, H. (1994). Learning about the gynecological health of women. In The policy series in reproductive health, No 2. The Population Council, Egypt.

Younis, N., Khattab, H., Zurayk, H., El-Mouelhy, M., Amin, M. F., \& Farag, A. M. (1993). A community study of gynecological and related morbidities in rural Egypt. Studies in Family Planning, 24, 175-186.

Zaman, R. (1996). Hakims and Vaids: The most popular doctors in Bangladesh, but they know little about AIDS. Aids Analysis Asia, 2(1), 12-123. 